\title{
P02-I2. Bupivacaine, a local anaesthetic, enhances immunogenicity of a multiepitopic DNA vaccine containing HIV promiscuous CD4 T cell epitopes
}

\author{
DS Rosa*, SP Ribeiro, EC Mairena, J Kalil and E Cunha-Neto
}

Address: Division of Clinical Immunology and Allergy, Dept. of Internal Medicine, University of São Paulo School of Medicine, Sao Paulo, Brazil

* Corresponding author

from AIDS Vaccine 2009

Paris, France. 19-22 October 2009

Published: 22 October 2009

Retrovirology 2009, 6(Suppl 3):PI7 doi:10.1 I86/1742-4690-6-S3-PI7

This abstract is available from: http://www.retrovirology.com/content/6/S3/PI7

(c) 2009 Rosa et al; licensee BioMed Central Ltd.

\section{Background}

DNA vaccines offer several advantages over other vaccine concepts, but poor immunogenicity in clinical use is still a general concern. Therefore, strategies to optimize the immunogenicity of DNA vaccines are urgently needed. Here, we explored the efficacy of Bupivacaine on augmenting the immunogenicity of a DNA vaccine encoding multiple HIV epitopes designed by our group.

\section{Methods}

The nucleotide sequence encoding 18 CD4/CD8 HIV-1 T cell epitopes was subcloned in pVAX-1. The DNA vaccine (pVAX-HIVBr18) or empty pVAX were used to immunize $\mathrm{BALB} / \mathrm{c}$ mice, alone or in the presence of Bupivacaine (a local anaesthetic with adjuvant properties). $\mathrm{T}$ cell responses were assessed by IFN-gamma and IL-2 ELISPOT, polyfunctional flow cytometry, Cytometric bead array on culture supernatants, and CFSE proliferation. Breadth of immune response was evaluated using ELISPOT assay against individual peptides.

\section{Results}

Coadministration of pVAXHIVBr18 with Bupivacaine was able to induce higher numbers of IFN-gamma secreting cells (ELISPOT) as well as a marked increase in IFNgamma and TNF- $\alpha$ secretion against pooled HIV peptides when compared to pVAXHIVBr18 alone. Additionally, coadministration with Bupivacaine induced an increase of trifunctional (IFN+/IL-2+/TNF+) CD4 T cells compared to the DNA vaccine alone. Also, coadministration of DNA vaccine with Bupivacaine increased IFN-gamma ${ }^{+} /$IL-2 $^{+}$ effector memory CD4 T cells and IFN-gamma producing effector memory CD8 T cells. Proliferative capacity of antigen-specific CD8 T cells was improved by Bupivacaine coadministration, as compared to DNA vaccine alone. This adjuvanted formulation induced multiepitopic responses with similar breadth as DNA alone.

\section{Conclusion}

Our data suggest that Bupivacaine can increase the magnitude of cytokine-producing effector memory $\mathrm{CD}^{+}$and $\mathrm{CD}^{+} \mathrm{T}$ cells, as well as increasing polyfunctional cytokine production of $\mathrm{CD} 4{ }^{+} \mathrm{T}$ cells, and increase proliferation of $\mathrm{CD}^{+} \mathrm{T}$ cells. This may have an impact in the clinical use of DNA vaccines. 